\title{
THE EULER CHARACTERISTIC OF A GENERIC WAVEFRONT IN A 3-MANIFOLD
}

\author{
S. IZUMIYA AND W. L. MARAR
}

(Communicated by Frederick R. Cohen)

\begin{abstract}
We give a relation between Euler characteristics of a generic closed Legendrian surface and its wavefront.
\end{abstract}

\section{INTRODUCTION}

In this note we shall compute the Euler characteristic of a generic wave front in a 3-manifold.

Let $N$ be a $(2 n+1)$-dimensional smooth manifold and $K$ be a contact structure on $N$ (i.e., $K$ is a nondegenerate tangent hyperplane field on $N$ ). An immersion $i: L \rightarrow N$ is said to be Legendrian if $\operatorname{dim} L=n$ and $d i_{x}\left(T_{x} L\right) \subset$ $K_{x}$ for any $x \in L$. We say that a smooth fibre bundle $\pi: E \rightarrow M$ is Legendrian if its total space $E$ is furnished with a contact structure and its fibres are Legendrian submanifolds. For a Legendrian immersion $i: L \rightarrow E, \pi \circ i: L \rightarrow$ $M$ is called a Legendrian map and the image of the Legendrian map $\pi \circ i$ is called the wavefront of $i$. It is denoted by $W(i)$.

From now on, we only consider the case of $n=2$. Then it is known that a generic wavefront has (semicubic) cuspidal edges $\left(A_{2}\right)$, swallowtails $\left(A_{3}\right)$, and points of transversal self-intersection $\left(A_{1} A_{1}, A_{1} A_{2}, A_{1} A_{1} A_{1}\right)$ as singularities [1] (see Figure 1 on the next page). We shall refer to the $A_{1} A_{1} A_{1}$-type point as a triple point of $i$.

If $L$ is a closed surface, then the number of swallowtails and triple points are finite. Our main result is the following :

Theorem. Let $i: L \rightarrow E$ be a generic Legendrian immersion of a closed surface. Then we have

$$
\chi(W(i))=\chi(L)+T(i)+\frac{S(i)}{2},
$$

where $\chi(X)$ is the Euler characteristic of $X, T(i)$ is the number of triple points on $W(i)$, and $S(i)$ is the number of swallowtails.

We remark that the corresponding result for a generic wavefront in a 2manifold is easily verified and that $\chi(W(i))=-d(i)$, where $d(i)$ denotes the number of double points on $W(i)$.

Received by the editors December 23, 1991.

1991 Mathematics Subject Classification. Primary 58C27.

Key words and phrases. Characteristics, wavefronts. 


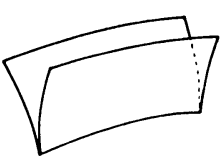

$A_{2}$

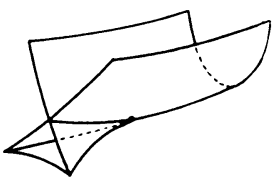

$A_{3}$

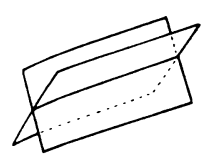

$A_{1} A_{1}$

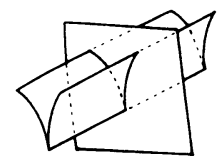

$A_{1} A_{2}$

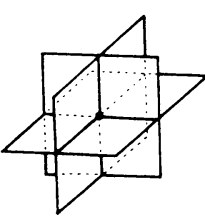

$A_{1} A_{1} A_{1}$

Figure 1

On the other hand, we consider an equidistant surface of a closed surface in $\mathbb{R}^{3}$. If the distance is sufficiently small, then the equidistant surface is diffeomorphic to the original surface. However, for some distances, singularities may appear in the equidistant surface and it may not be homeomorphic to the original surface. Hence, it is interesting to study the relation between topologies of the equidistant surface and of the original surface. These subjects are studied in the theory of Legendrian singularities. In fact, it is known that singularities of equidistant surfaces are locally diffeomorphic to singularities of wavefronts (see [1]). Then we show the general property of the Euler characteristic of global wavefronts.

In order to prove the theorem, we shall apply the method which has been introduced to compute the Euler characteristic of the image of a stable perturbation of an $\mathscr{A}$-finite map germ in [2].

All maps considered here are differentiable of class $C^{\infty}$ unless stated otherwise.

\section{Proof of The theorem}

In this section we shall give a proof of the theorem. Let $i: L \rightarrow E$ be a generic Legendrian immersion of a closed surface. Since the Euler characteristic is a topological invariant, we can ignore cuspidal edges. We now define the following sets:

$$
\begin{aligned}
& D^{2}(i)=\operatorname{cl}\left\{x \in L \mid \sharp(\pi \circ i)^{-1} \pi \circ i(x) \geq 2\right\}, \\
& D^{3}(i)=\left\{x \in D^{2}(i) \mid \sharp(\pi \circ i)^{-1} \pi \circ i(x)=3\right\}, \\
& D^{2}(i,(2))=\left\{x \in D^{2}(i) \mid \sharp(\pi \circ i)^{-1} \pi \circ i(x)=1\right\},
\end{aligned}
$$

where $\operatorname{cl} X$ is the topological closure of $X$. Then we have the following diagram:

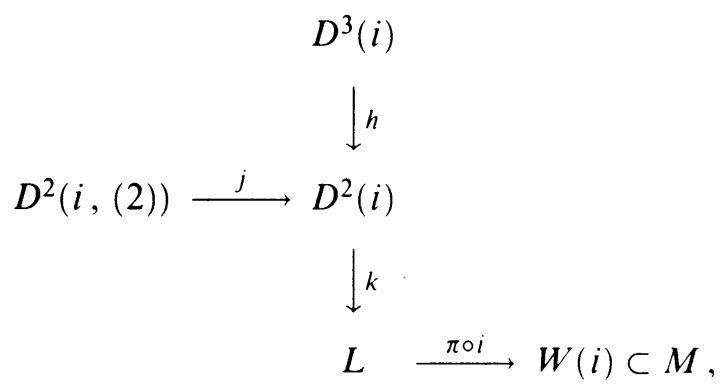

where $h, j$, and $k$ are inclusions. 
By the characterization of generic wavefronts (see [1]), $D^{2}(i)$ is a union of curves on $L$ with self-intersection and circles, $D^{3}(i)$ is the inverse image of triple points, and $D^{2}(i,(2))$ is the set of swallowtails of $\pi \circ i$. It follows that these are immersed submanifolds of $L$ with $\operatorname{dim} D^{2}(i)=1$ and $\operatorname{dim} D^{3}(i)=$ $\operatorname{dim} D^{2}(i,(2))=0$.

In order to prove the theorem, we need the following formula.

Lemma 1.1. $\chi(W(i))=\chi(L)-\frac{1}{2} \chi\left(D^{2}(i)\right)+\frac{1}{2} \chi\left(D^{2}(i,(2))\right)-\frac{1}{6} \chi\left(D^{3}(i)\right)$.

Proof. Consider the equation

$$
\chi(W(i))=\alpha \chi(L)+\beta \chi\left(D^{2}(i)\right)+\gamma \chi\left(D^{2}(i,(2))\right)+\delta \chi\left(D^{3}(i)\right),
$$

where $\alpha, \beta, \gamma$, and $\delta$ are unknown variables. We solve this by a purely combinatorial method.

We now construct a triangulation $K_{i}$ of the stratified set $W(i)$ as follows: We start to triangulate $W(i)$ by including the image of $D^{2}(i,(2))$ and the image of $D^{3}(i)$ among the vertices of $K_{i}$. After this, we build up the oneskeleton $K_{i}^{(1)}$ of $K_{i}$ so that the image of $D^{2}(i)$ is a subcomplex of $K_{i}^{(1)}$. We complete our procedure by constructing the two-skeleton $K_{i}^{(2)}$.

Since $\pi \circ i$ and its restrictions to $D^{2}(i), D^{2}(i,(2))$, and $D^{3}(i)$ are proper and finite-to-one mappings, we can pull back $K_{i}$ to obtain a triangulation for $L, D^{2}(i), D^{2}(i,(2))$, and $D^{3}(i)$. Let $C_{j}^{X}$ be the number of $j$-cells in $X$, where $X=W(i), \quad L, \quad D^{2}(i), \quad D^{2}(i,(2))$, or $D^{3}(i)$. Then the equation $(*)$ can be written as

$$
\begin{aligned}
\sum_{j}(-1)^{j} C_{j}^{W(i)}= & \alpha \sum_{j}(-1)^{j} C_{j}^{L}+\beta \sum_{j}(-1)^{j} C_{j}^{D^{2}(i)} \\
& +\gamma \sum_{j}(-1)^{j} C_{j}^{D^{2}(i,(2))}+\delta \sum_{j}(-1)^{j} C_{j}^{D^{3}(i)},
\end{aligned}
$$

where $C_{j}^{X}=0$ if $i>\operatorname{dim} X$. So, if we can find real numbers $\alpha, \beta, \gamma$, and $\delta$ such that

$$
C_{j}^{W(i)}=\alpha C_{j}^{L}+\beta C_{j}^{D^{2}(i)}+\gamma C_{j}^{D^{2}(i,(2))}+\delta C_{j}^{D^{3}(i)},
$$

for any $j$, then we have solutions of the equation $(*)$. By the construction of the triangulation, we may concentrate on solving $(* *)$ in the case when $j=0$. We remark that $\pi \circ i$ is 3-to-1 over the points in the image of $D^{3}(i), 1$-to-1 over the points in the image of $D^{2}(i,(2)), 2$-to-1 over the points in the image of $D^{2}(i)-\left(D^{2}(i,(2)) \cup D^{3}(i)\right)$, and 1-to-1 over the points in the image of $S-D^{2}(f)$. It follows that the equation

$$
C_{0}^{W(i)}=\alpha C_{0}^{L}+\beta C_{0}^{D^{2}(i)}+\gamma C_{0}^{D^{2}(i,(2))}+\delta C_{0}^{D^{3}(i)}
$$

is equivalent to the system of linear equations

$$
\left(\begin{array}{l}
1 \\
1 \\
1 \\
1
\end{array}\right)=\left(\begin{array}{llll}
1 & 0 & 0 & 0 \\
2 & 2 & 0 & 0 \\
1 & 1 & 1 & 0 \\
3 & 3 & 0 & 3
\end{array}\right)\left(\begin{array}{l}
\alpha \\
\beta \\
\gamma \\
\delta
\end{array}\right) .
$$

We can easily solve this equation, so that $\alpha=1, \beta=-1 / 2, \gamma=1 / 2$, and $\delta=-1 / 6$. This completes the proof. 
Then we can prove the theorem.

Proof of the theorem. By the definition we have $\chi\left(D^{2}(i,(2))\right)=S(i)$ and $\chi\left(D^{3}(i)\right)=3 T(i)$. Since $D^{2}(i)$ is a union of closed curves on the surface $L$ with $3 T(i)$ crossings, then we can triangulate it with $3 T(i)+n$-cells and $6 T(i)+n$ 1-cells, where $n$ is the number of circles in $D^{2}(i)$. It follows that $\chi\left(D^{2}(i)\right)=-3 T(i)$. If we substitute these in the formula in Lemma 1.1, then we have

$$
\chi(W(i))=\chi(L)+T(i)+\frac{1}{2} S(i) .
$$

This completes the proof of the theorem.

\section{ACKNOWLEDGMENT}

This work was done during the authors' stay at the University of Liverpool. The authors would like to thank the Department of Pure Mathematics. The first author acknowleges the financial support of JSPS and the second author the financial support of CAPES.

\section{REFERENCES}

1. V. I. Arnol'd, Singularities of caustics and wavefronts, Math. Appl. (Soviet Ser.), vol. 62 , Kluwer Academic, Dordrecht, 1990.

2. W. L. Marar, The Euler characteristic of the disentanglement of the image of a corank 1 map germ, Lecture Notes in Math., vol. 1462, Springer, Berlin and New York, 1991, pp. $212-220$.

Department of Mathematics, Faculty of Science, Hokkaido Univeristy, Sapporo 060. JAPAN

Instituto de Ciências Matemáticas de São Carlos, Universidade de São Paulo, Caixa Postal 668, 13560-São CARLos-(SP)-Brazil 